\title{
Dibujar la conversación en ELE: la enseñanza de la toma de turnos del español
}

\author{
GUADALUPE ESPINOSA GUERRI \\ Universitat de València \\ guadalupe.epinosa@uv.es
}

\begin{abstract}
Resumen: Uno de los componentes necesarios para el desarrollo de la competencia pragmática es la toma de turnos. No obstante, aunque nuestro conocimiento sobre la estructura de la conversación coloquial y sobre el funcionamiento del sistema de toma de turnos se ha visto ampliado gracias a los estudios que se han realizado desde el Análisis de la Conversación y el Español Coloquial, son muy pocos los intentos de trasvasar ese conocimiento teórico al campo de la enseñanza de lenguas extranjeras (Cestero, 2012).

El presente trabajo pretende dar a conocer algunos de los hallazgos en torno al sistema de alternancia de turnos de la conversación coloquial española y proponer dos herramientas didácticas para su enseñanza en el aula de ELE: 1) los dientes de sierra, herramienta de visualización que permite reconocer gráficamente la relación entre los turnos de un diálogo; y 2) una tipología de figuras interaccionales que recoge las diferentes estructuras que se producen en la toma de turnos de la conversación coloquial española.
\end{abstract}

Palabras clave: competencia conversacional, competencia pragmática, alternancia de turnos, estructuras interaccionales, figuras, conversación coloquial.

\section{Drawing conversations in ELE: the teaching of the Spanish turn-taking system}

Abstract: One of the necessary components for the development of the pragmatic competence is the turn-taking system. Although our knowledge of the natural conversation structure and the functioning of the turn-taking system has been expanded thanks to the Conversation Analysis and Colloquial Spanish studies, there are very few attempts to transfer that theoretical knowledge to the field of foreign language teaching (Cestero, 2012).

The aim of this paper is to present some of the findings about the turn-taking system of the Spanish natural conversations and to propose two didactic tools for their teaching in Spanish courses: 1) the Sawteeth, a visualization tool that allows to graphically recognize the relationship between the turns of a dialogue; and 2) a typology of interactional figures that includes the different structures that take place in the turn-taking system of the Spanish natural conversation.

Keywords: conversational competence, pragmatic competence, turn-taking system, interactional structures, figures, natural conversation.

\section{La enseñanza de la toma de turnos}

Si la conversación coloquial es la forma más básica de comunicación de cualquier lengua (Briz and Grupo Val.Es.Co, 2003; Schegloff, 2007; Heritage, 2013; Mancera, 2016), debería tener este género un lugar central en la enseñanza de lenguas extranjeras. No obstante, varios autores han advertido de su escasa presencia en los manuales y en las 
clases de Español como Lengua Extranjera (ELE) o de la falta de autenticidad de las muestras de habla que se emplean para enseñar los diferentes rasgos de la conversación coloquial.

Cestero Mancera (2012) señala la falta de propuestas didácticas encaminadas a trabajar las características conversacionales en los manuales de ELE. Según esta autora, la enseñanza de los fenómenos, mecanismos y elementos conversacionales debe ser explícita, pero integrada con otros contenidos gramaticales, léxicos, pragmáticos y nociofuncionales. Los estudios que han explorado las conversaciones de estudiantes de español evidencian dificultades en el manejo de diferentes recursos conversacionales, como los turnos de apoyo, la interpretación de los lugares de transición pertinente (LTP) o el desarrollo temático (Cestero Mancera, 2016).

Padilla García (2012) analiza las muestras de diálogos coloquiales de varios manuales de ELE y llega a la conclusión de que

los diálogos de ELE y las conversaciones coloquiales no son fenómenos completamente distintos. Ambos comparten unidades y utilizan mecanismos conversacionales parecidos. [...] No obstante, todos los diálogos didácticos analizados, independientemente del enfoque que elijan, se alejan de las conversaciones coloquiales en todo aquello que afecta a lo más propiamente conversacional y coloquial, es decir, a lo más dinámico y creativo. (Padilla García, 2012: 102)

«Lo más propiamente conversacional y coloquial» es la energeia discursiva o dinamismo discursivo, es decir, la lucha por el turno, los solapamientos, la variedad de tópicos y la presencia frecuente de intervenciones reactivo-iniciativas. Mientras que rasgos coloquiales como la temática no especializada, la relación vivencial de proximidad o el léxico coloquial son rasgos que se plasman fielmente en los diálogos didácticos, los rasgos propios del dinamismo discursivo no se tienen en cuenta, de modo que se suelen crear diálogos «encorsetados, estáticos y poco reales» (Padilla García, 2012: 102).

García García (2016) señala como posible causa de la escasa presencia de la conversación coloquial en el aula de español la creencia generalizada de que la toma de turnos de la conversación coloquial no se puede enseñar por no estar preestablecida, como sí sucede en otros géneros más formales, como la entrevista, el debate, etc. Esta creencia es la que explica que la conversación coloquial haya sido un género poco trabajado por la Lingüística tradicional, que consideraba la conversación como un género carente de estructura, por lo que su estudio no se podía sistematizar. Esto retrasó el estudio de lo coloquial hasta mediados del siglo XX. Los estudios surgidos dentro del marco del Análisis de la Conversación proporcionan la base teórica necesaria para entender las normas, estructuras y rasgos que forman parte de la conversación coloquial y dan muestras de sus peculiaridades frente a otros géneros y registros.

También apunta esta autora, como posible factor, la creencia según la cual los hablantes extranjeros ya dominan los rasgos propios de la toma de turnos de la conversación coloquial de su lengua de origen, de modo que no es necesario enseñarlos. Esta creencia se fundamenta en la idea de que el sistema de alternancia de turnos es universal. Si bien Stivers et al. (2009) defienden que la alternancia de turnos es uno de los universales lingüísticos, también reconoce que las posibles variantes culturales son de carácter fundamentalmente cuantitativo: "Our empirical evidence suggests robust human universals in this domain [la alternancia de turnos], where local variations are quantitative only, pointing to a single shared infrastructure for language use...» (Stivers et al., 2009: 
10587). De este modo, todas las lenguas estudiadas por este grupo muestran una tendencia general a evitar el solapamiento y a minimizar el silencio entre los turnos conversacionales. La diferencia más relevante afecta al tiempo de transición entre un turno y otro, que varía en un rango de $250 \mathrm{~ms}$. En resumen, según estos autores, hay una parte sustancial de la alternancia de turnos que es común a todas las lenguas, y sobre ella se sustentan otros rasgos específicos que varían (sobre todo cuantitativamente) entre las culturas.

Lo cierto es que los avances en el campo del Análisis de la Conversación sobre la alternancia de turnos ofrecen los conocimientos teóricos suficientes como para ayudar a desarrollar la competencia conversacional de los alumnos de español de forma más completa. No obstante, el trasvase de conocimientos a la didáctica de lenguas ha sido muy escaso:

Los conocimientos que tenemos en la actualidad acerca del funcionamiento de la conversación española son bastante completos, por lo que es posible diseñar planes curriculares que atiendan a contenidos conversacionales; sin embargo, son muy pocos los trabajos realizados con respecto a diferencias culturales en la estructuración de la conversación y a las características que presenta la conversación en LE, así como a la enseñanza de mecanismos y fenómenos conversacionales. (Cestero Mancera, 2012: 31)

La falta de conexión entre la investigación lingüística y la didáctica de lenguas no se limita solo al ámbito de la conversación coloquial y la toma de turnos. Se trata de un hecho generalizado que afecta a casi todas las ramas de la Lingüística. Tal y como señala Padilla García (2012), la falta de comunicación entre lingüistas y creadores de materiales puede ser uno de los factores que afectan a la falta de representatividad realista de la conversación coloquial en los materiales didácticos. Reyes Llopis también apunta la existencia de una brecha entre la lingüística teórica y sus aplicaciones prácticas al aula de lengua (Herrera, 2013).

El presente trabajo pretende dar a conocer algunos de los hallazgos en torno a la alternancia de turnos y plantear cómo se podrían trabajar en las clases de español a través de dos herramientas: 1) los dientes de sierra, herramienta de visualización que permite reconocer gráficamente la relación entre los turnos de un diálogo; y 2) una tipología de figuras interaccionales que recoge las diferentes estructuras que se producen en la toma de turnos de la conversación coloquial española.

\section{Dientes de sierra: una herramienta para visualizar la toma de turnos}

Los dientes de sierra son una herramienta creada por el grupo Val.Es.Co. (Briz, 2006; Espinosa-Guerri, 2016) para la representación visual de la relación entre las unidades superiores del discurso dialogal, esto es, para observar la forma en que los hablantes establecen conexiones entre su turno y aquellos que se sitúan por delante y por detrás de este. Presenta tres características fundamentales (Espinosa-Guerri y García-Ramón, 2017): es 1) sistemática, en tanto que contiene unos símbolos básicos y se rige por unas reglas de uso fijadas; 2) exhaustiva; de modo que con ella se puede analizar cualquier intervención de un discurso dialogal sin dejar residuos; y, 3) aplicable a cualquier discurso dialogal con el objetivo de observar las estructuras interaccionales, medirlas y 
compararlas, tanto entre géneros distintos, como entre grupos de hablantes, situaciones comunicativas, etc. Los símbolos básicos de la herramienta son cinco (ver Tabla 1).

\begin{tabular}{|c|c|c|c|c|}
\hline \multicolumn{3}{|c|}{ simBolos } \\
\hline $\begin{array}{c}\text { Intervención } \\
\text { iniciativa }\end{array}$ & $\begin{array}{c}\text { Intervención } \\
\text { reactiva }\end{array}$ & Intercambio & $\begin{array}{c}\text { Intervención } \\
\text { discontinua }\end{array}$ & $\begin{array}{c}\text { Intervención } \\
\text { independiente }\end{array}$ \\
\hline & & $\ldots . .$. & \\
\hline
\end{tabular}

Tabla 1. Símbolos básicos de los dientes de sierra.

Una intervención iniciativa es una intervención que «provoca una reacción posterior» (Grupo Val.Es.Co., 2014: 17). Una intervención reactiva es una «reacción a una intervención previa» (2014: 17). Un intercambio está constituido por «dos intervenciones sucesivas de distintos emisores, una de inicio y otra de reacción, constituyen la mínima unidad dialógica estructural»(2014: 23). Una intervención discontinua es aquella atribuida a un emisor pero que ha sufrido algún tipo de interrupción a lo largo de su emisión (2014: 20), por ejemplo, con risas del interlocutor o con turnos de apoyo como $m m$, ajá, claro, etc. Por último, las intervenciones independientes son aquellas que «no reaccionan a ninguna intervención previa ni generan reacciones posteriores» (EspinosaGuerri, 2016).

A continuación, se explica cuál es el método empleado para combinar los símbolos básicos presentados previamente. Ante un fragmento de conversación como el de (1), seguimos estos tres pasos.

$(1)^{1}$

$\mathrm{A} 1^{2}$ : y ella aquí no tiene familia ¿no?

B1: no

A2: ¿y con quién vive? // ¿con compañeras de piso?

B2: con tres compañeras de piso

En primer lugar, nos preguntamos qué intervención o intervenciones reaccionan a la primera intervención, A1. Una vez identificamos la intervención B2 como reacción a A1 dibujamos el símbolo de intervención iniciativa ubicando el punto de inicio a la altura de A1 (ver;Error! No se encuentra el origen de la referencia.) y la unimos con el símbolo de intervención reactiva ubicando el final de esta a la altura de la intervención B2 (ver 3). El resultado es un diagrama en forma de pico que representa, tal y como hemos indicado en el cuadro de símbolos (ver Tabla 1), un intercambio básico, es decir, la unión de una intervención iniciativa y una reactiva. Este esquema constituye la unidad mínima de

\footnotetext{
${ }^{1}$ Para la trascripción de los fragmentos de conversaciones coloquiales empleamos los signos del grupo Val.Es.Co. (ver Grupo Val.Es.Co., 2014).

${ }^{2}$ De aquí en adelante, identificaremos las intervenciones con un número a la derecha de la inicial que identifica al interlocutor: A1 indica la primera intervención del interlocutor A, B3 la tercera intervención del interlocutor B, y así sucesivamente.
} 
cualquier diálogo, es lo que se conoce en el Análisis de la Conversación como par adyacente.

(2)

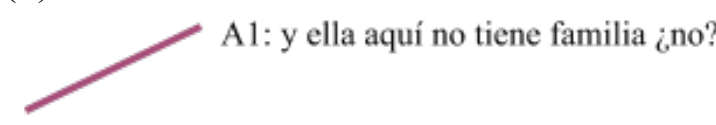

B1: no

A2: ¿y con quién vive? // ¿con compañeras de piso?

B2: con tres compañeras de piso

(3)

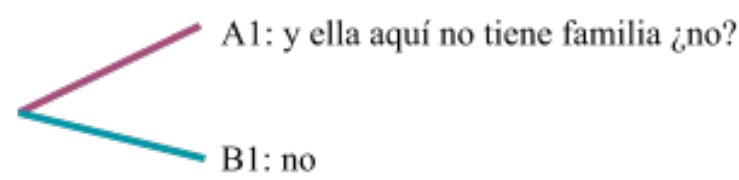

A2: ¿y con quién vive? // ¿con compañeras de piso?

B2: con tres compañeras de piso

Una vez hecho esto, repetimos el proceso: nos preguntamos qué intervención o intervenciones posteriores reaccionan a B1 y las unimos. Puesto que en este fragmento $\mathrm{A} 2$ es la reacción a $\mathrm{B} 1$, continuamos el diagrama tal y como se ve en (4).

(2)

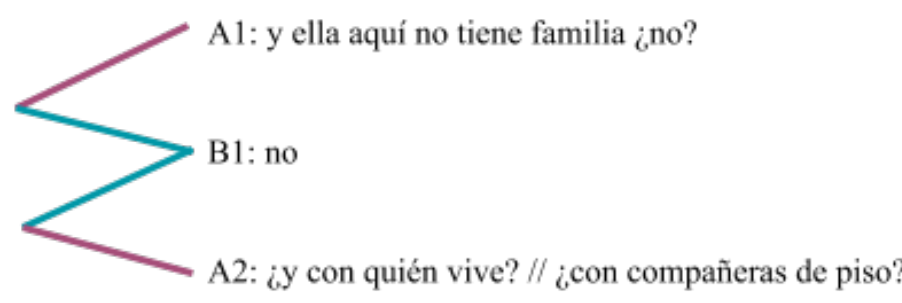

B2: con tres compañeras de piso 
De nuevo, repetiríamos el proceso con las dos últimas intervenciones. La intervención B2 es reacción a A2, de modo que las unimos de la misma forma que las intervenciones previas (ver (5). Como se puede observar, el esquema resultante es un diagrama en zigzag que representa una sucesión de pares adyacentes encadenados.

(3)

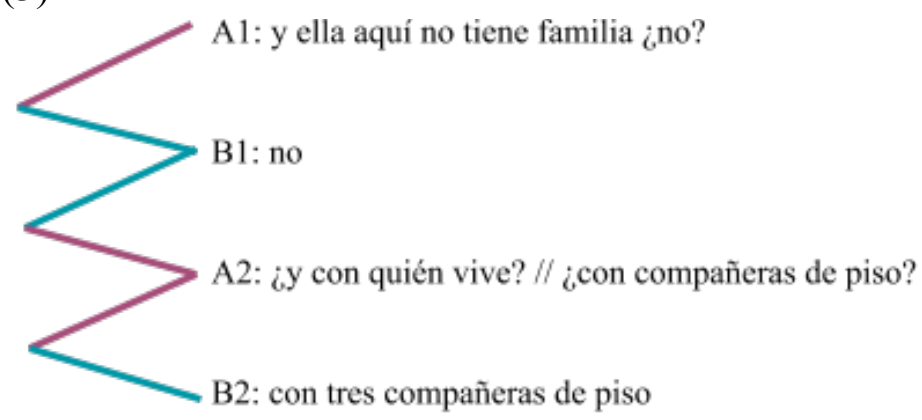

Si en lugar de tener intervenciones completas como en las del ejemplo anterior, nos encontramos con intervenciones discontinuas, el esquema cambia. Ante un ejemplo como el de (4), vemos que la intervención A1 está formada por tres segmentos o cambios de voz debido a las interrupciones solapadas del hablante B. Estas interrupciones de B, sin embargo, no detienen la intervención A1 porque no le roban el turno. Así pues, la intervención completa de A1 es: «[bueno] pues por lo visto era curioso porque en el norte (RUIDO) erraa- era al revés ¿no?/ y yo [me-] todos menos él/ me han debido ver la cara diciendo ¿y con quién? [(RISAS)] debe haber/// yy me señala una y deice/ ${ }^{\circ}$ (este es del norte $)^{\circ} \gg$.

(4)

A1: [bueno] pues por lo visto era curioso porque en el norte (RUIDO) eraa-

era al revés ¿no?/ y yo [me-]

B1: $\quad$ [claro]

A1: todos menos él / me han debido ver la cara diciendo ¿y con quién?

[(RISAS)]

B2: [(RISAS)]

A1: debe haber /// yy me señala una y dice $/{ }^{\circ}(\text { este es del norte })^{\circ}$

Para visualizar este fragmento, seguimos los siguientes pasos: puesto que la intervención de A1 es una intervención iniciativa, añadimos el símbolo de intervención iniciativa (ver (8). 
(6)

A1: [bueno] pues por lo visto era curioso porque en el norte (RUIDO) eraaera al revés ¿no?/ y yo [me-]

B1:

[claro]

A1: todos menos él / me han debido ver la cara diciendo ¿y con quién?

[(RISAS)]

B2: [(RISAS)]

A1: debe haber $/ / /$ yy me señala una y dice $/{ }^{\circ}(\text { este es del norte })^{\circ}$

Puesto que A1 es discontinua y llega hasta «debe haber...», se alarga la línea de la intervención iniciativa de A1 hasta el final de la intervención y se indica con una línea discontinua que ha sido interrumpida (ver(7).

(5)

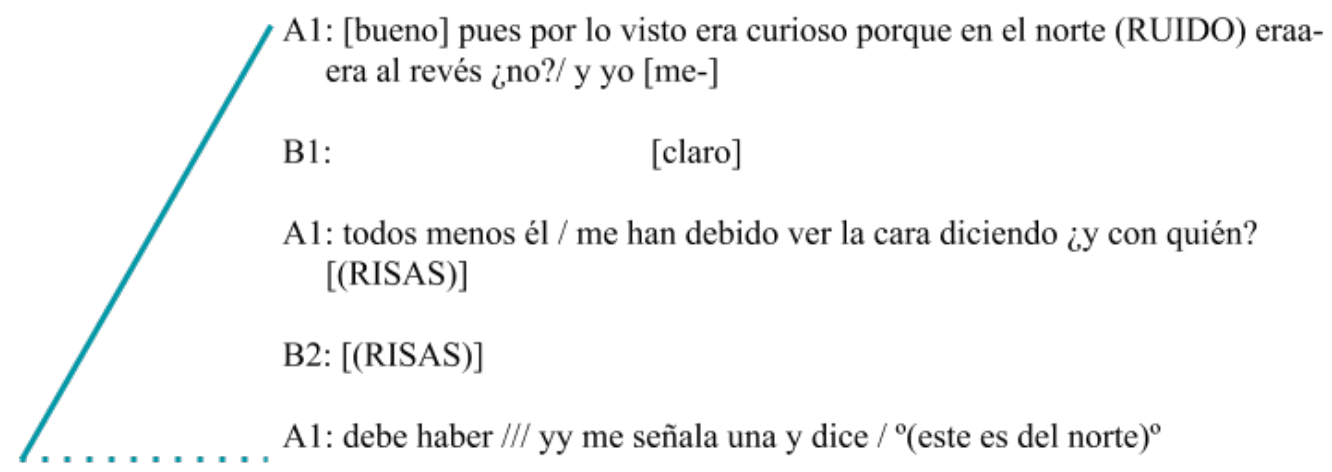

Hecho esto, señalamos con el símbolo de intervención reactiva las dos intervenciones B1 y B2 (9).

(7)

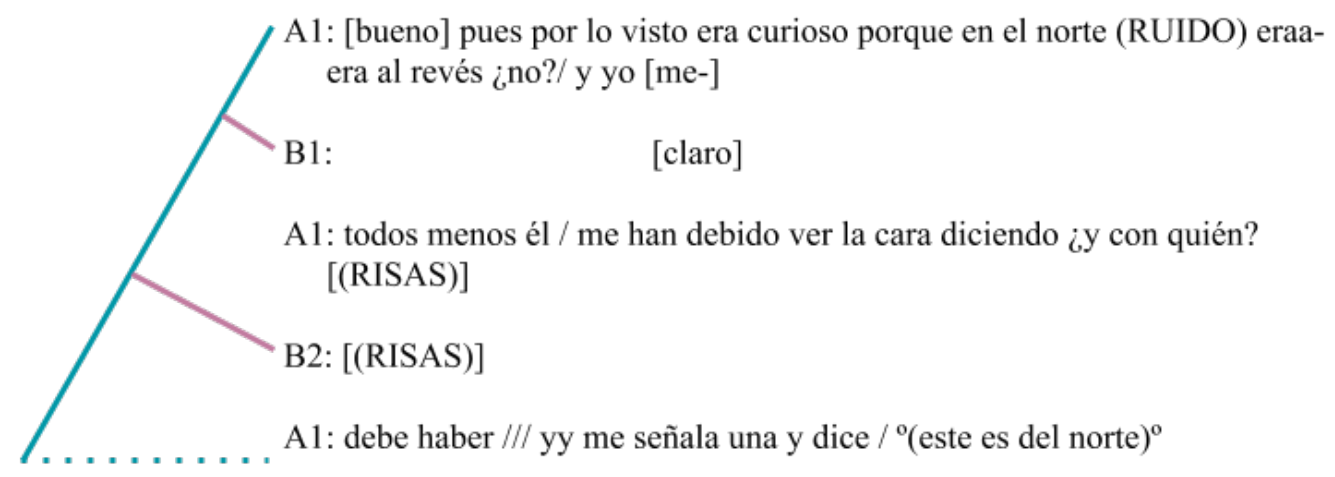




\section{Tipología de figuras conversacionales}

A partir de esta herramienta de visualización realizamos un estudio previo en el cual nos propusimos observar cuáles eran los patrones interaccionales que nos encontramos en este nivel de la estructura de los discursos dialógicos para, posteriormente, clasificarlos en una tipología (Espinosa-Guerri y García-Ramón, 2019). Con este objetivo aplicamos los dientes de sierra a un corpus de entrevistas y conversaciones y el resultado fue una tipología de nueve estructuras interaccionales a las cuales llamamos figuras.

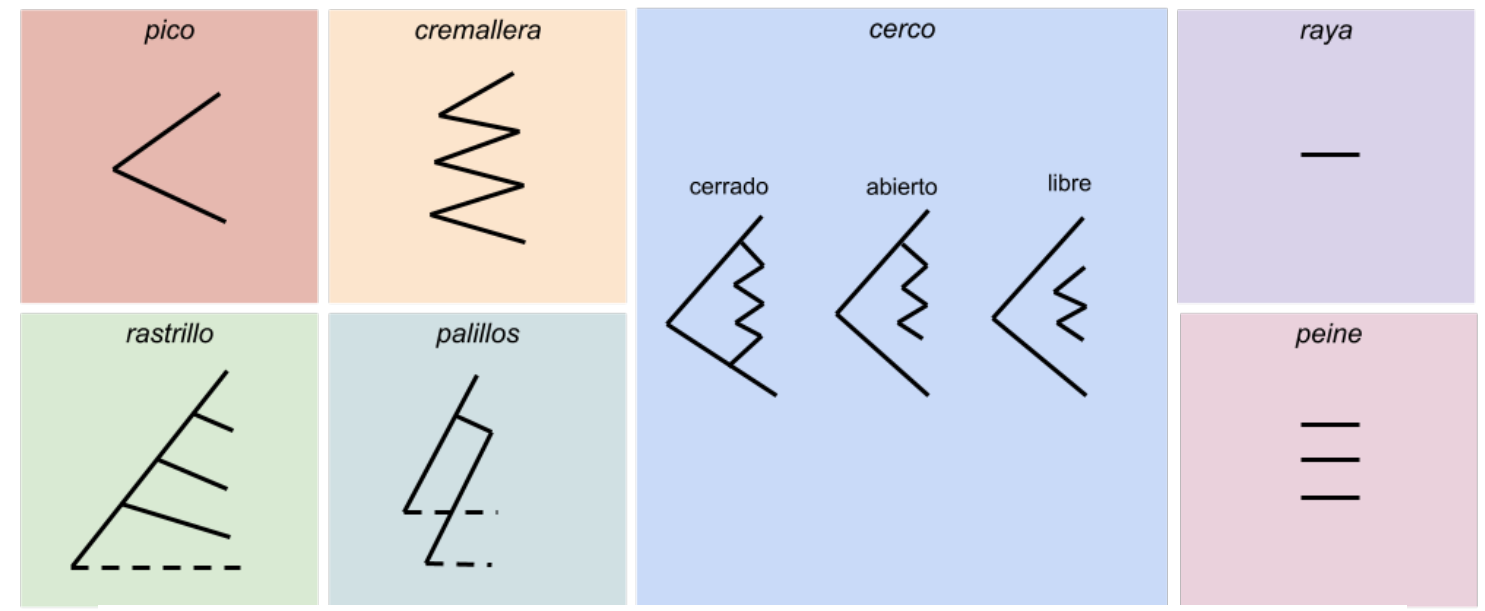

Tabla 2. Tipología de figuras interaccionales.

Estas figuras representan formas distintas que tenemos los hablantes de establecer conexiones con los turnos de habla de nuestros interlocutores. Cada una de ellas presenta unas características propias y una serie de funciones pragmáticas asociadas. A continuación, se detallará en qué consiste cada figura, qué información aporta sobre el rol interactivo de los participantes y a qué funciones pragmáticas están vinculadas.

\subsection{Pico y cremallera}

En el fragmento (10), se dan dos cremalleras y un pico. La cremallera es la figura que hemos visto en el ejemplo (5), la cual consiste en una sucesión de intercambios encadenados. Las intervenciones A1, B1 y A2 se unen a través de esta figura, pero en A3, el hablante no reacciona a B1, sino que inicia un nuevo tema de conversación indicando a $\mathrm{B}$ que se va a tumbar porque está cansada. B2 reacciona a dicha intervención, pero $\mathrm{A}$ no reacciona a B2, sino que inicia un nuevo tema, esta vez, sobre la programación de la televisión. Este tema se desarrolla gracias a la reacción de B3, A5 y las intervenciones sucesivas, creando una nueva cremallera. El intercambio que se da, en cambio, entre A3 y B2 es un intercambio aislado que constituye un diálogo mínimo en términos del grupo Val.Es.Co. ${ }^{3}$ A esta figura la llamamos pico.

\footnotetext{
${ }^{3}$ Según el sistema de unidades de Val.Es.Co. el diálogo es una «Unidad dialógica definible en términos estructurales, limitada prototípicamente por una intervención-turno iniciativa al inicio y por una intervención-turno reactiva al final de la misma» (Grupo Val.Es.CO., 2014:31).
} 
(8)

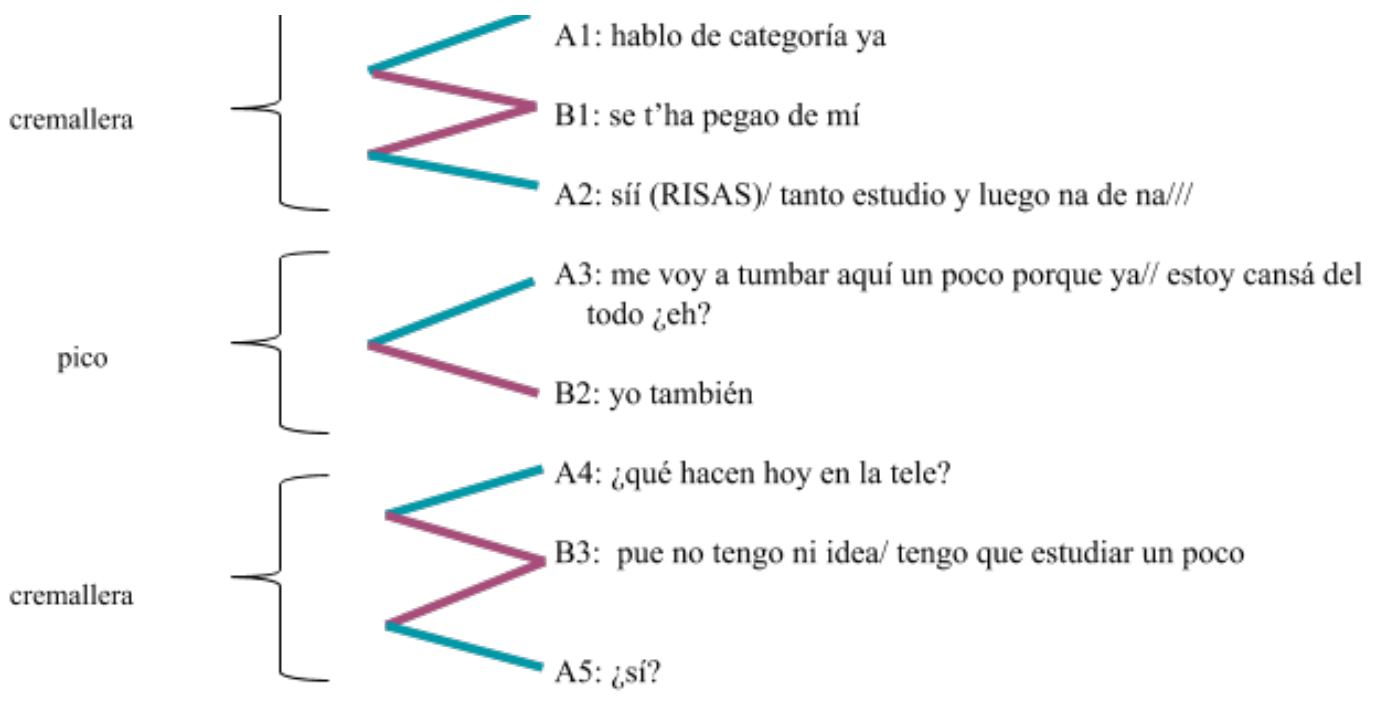

La participación de los emisores en el pico es equitativa, es decir, ninguno de los hablantes tiene un rol dominante desde el punto de vista interactivo. No obstante, es una figura muy escasa en la conversación coloquial española $(1,5 \%)$ y es más propia de otro tipo de géneros como la entrevista sociolingüística (Espinosa-Guerri y García-Ramón, 2017). Su presencia abundante fragmenta el diálogo y no facilita el desarrollo del tema de la conversación. Se suele emplear para solucionar algún tema secundario, tal y como se aprecia en el ejemplo anterior, o para responder a una pregunta breve.

En la cremallera, la participación de los hablantes también es equitativa, pero a diferencia del pico, es una figura muy abundante en la conversación coloquial $(37,8 \%)$, genera dinamismo conversacional, pues los hablantes se alternan el turno de forma continuada. Esto favorece el desarrollo de los temas, de modo que se emplea para aportar información o compartir opiniones sobre un tema, también para discutir sobre alguna cuestión polémica (de forma cooperativa) o para desarrollar algún aspecto del tema mediante preguntas y respuestas encadenadas.

Estas figuras no suelen presentar ningún problema para los estudiantes de español, que las ejecutan en sus conversaciones coloquiales de forma inconsciente y sin dificultades. El problema surge cuando las conversaciones de los estudiantes están formadas únicamente $o$ en un porcentaje excesivo por estas dos figuras. Las conversaciones dominadas por estos dos patrones crean una sensación de «interrogatorio», tal y como advierte (García García, 2016: 15-16) al analizar el siguiente fragmento de una conversación de alumnos alemanes, la cual hemos visualizado para detectar las figuras producidas por estos estudiantes: 
(9)

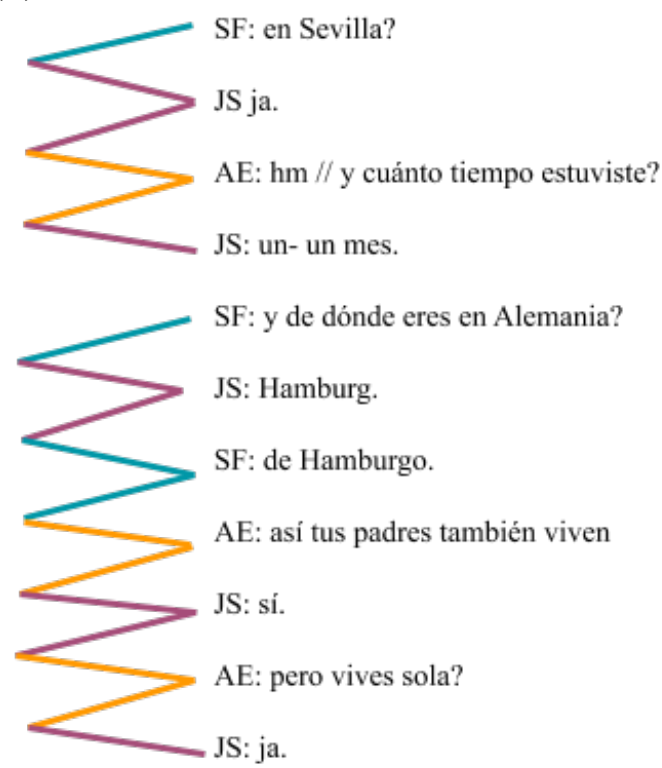

La conversación coloquial es un género mucho más variado desde el punto de vista de la interacción, los hablantes conectan sus turnos mediante otras estructuras más allá del pico y la cremallera. Una conversación coloquial dominada por estas dos figuras será un diálogo, pero no será una conversación coloquial prototípica del español. Por ello, conviene conocer y saber producir en español otras estructuras, así como sus implicaciones pragmáticas, ya que estas pueden variar en función de la cultura o de los rasgos situacionales ${ }^{4}$ (relación de igualdad entre los participantes, cotidianidad temática, fin interpersonal, etc.). También es importante que los diálogos que tratan de ejemplificar la conversación coloquial reflejen toda la variedad de figuras existente y que no se limiten, como suceder, a una sucesión de picos y cremalleras.

\subsection{Rastrillo y palillos}

Una de las figuras más representativas de la conversación coloquial española es el rastrillo. Esta figura se produce cuando el hablante A emite una intervención iniciativa discontinua, la cual es interrumpida, aunque no cortada, por turnos de apoyo del interlocutor $^{5}$ que muestran interés o acuerdo sobre lo dicho y animan al hablante $\mathrm{A}$ a continuar su turno. Esto es lo que sucede en (12).

\footnotetext{
${ }^{4}$ Para una descripción más detallada sobre los rasgos situacionales ver Briz Gómez (2010).

${ }^{5}$ Para una descripción detallada de los turnos de apoyo, consultar (Cestero Mancera, 2000).
} 


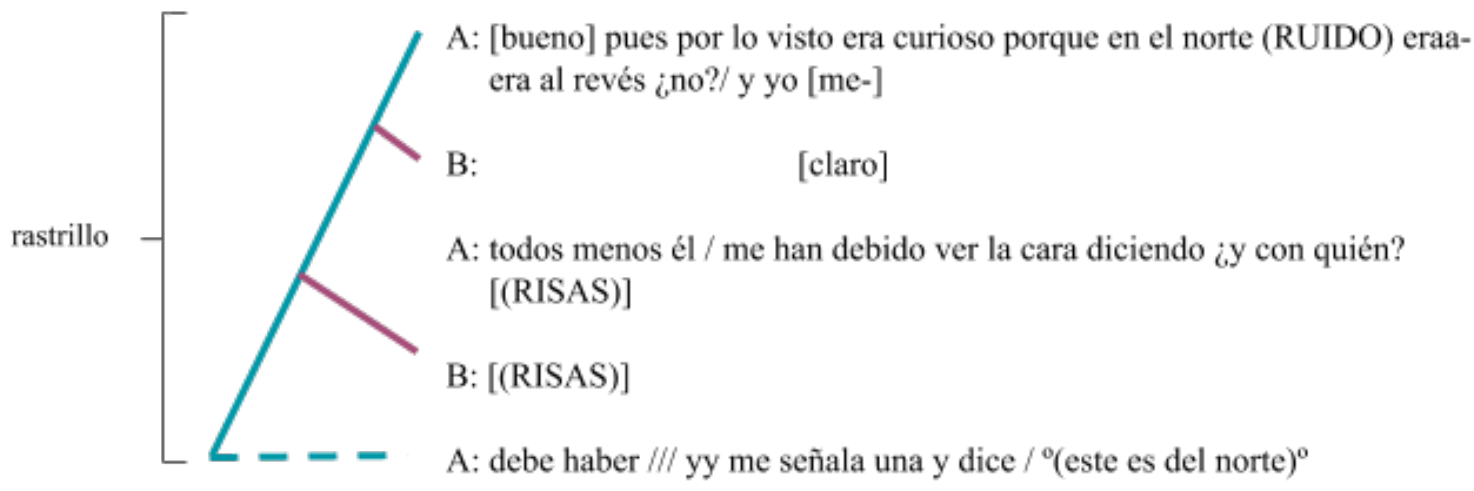

En la producción de esta figura, a diferencia de en las dos figuras previas, la participación de los interlocutores no es equitativa, sino que aquel que emite el turno principal (en este caso, la intervención de A) asume un rol dominante, mientras que el hablante que emite las intervenciones reactivas (B) tiene un papel más pasivo. Se trata de una figura muy abundante en la conversación coloquial, constituye el $41,1 \%$ del corpus. Las intervenciones reactivas pueden producirse de forma solapada (como en el ejemplo previo), pero no necesariamente. Los turnos de apoyo pueden tener una forma verbal (como el primero del ejemplo, claro) o no verbal (como el segundo, RISAS). Uno de los problemas que se han detectado en la alternancia de turnos de estudiantes de español es la transferencia de los turnos de apoyo propios de la lengua materna, tal y como se puede percibir en el caso de los hablantes alemanes del ejemplo (11) y como documenta Inglés Candelas (2016) en el caso de alumnos británicos. Esta estructura suele tener una finalidad cooperativa, porque los turnos de apoyo son muestras explícitas del interlocutor que, aunque pueden producirse de forma solapada, no pretenden robar el turno, sino mostrar interés, acuerdo, seguimiento, etc. No obstante, las intervenciones reactivas pueden no ser turnos de apoyo sino intentos de robo de turno, aunque esto es menos frecuente.

La figura palillos está relacionada con el rastrillo en tanto que está formada por dos intervenciones discontinuas paralelas, tal y como se aprecia en el ejemplo (13). 
(11)

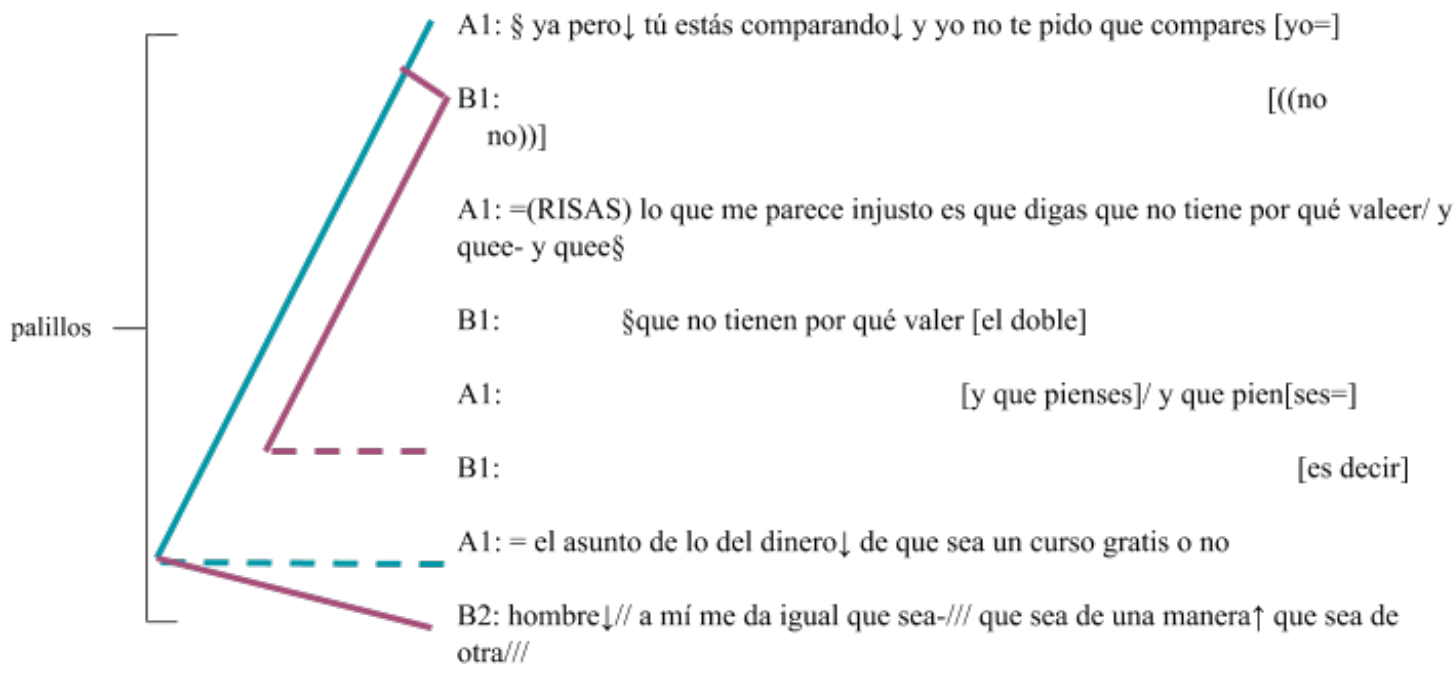

La figura se produce cuando el hablante A emite un turno y B reacciona a dicho turno, pero ninguno de los dos abandona su intervención, de modo que cada uno transmite su mensaje sin reaccionar a lo que dice el otro, hasta que uno de los dos consigue la reacción del interlocutor. Así pues, las dos intervenciones se podrían leer de forma continua eliminando la intervención del interlocutor sin que esto altere el sentido de la intervención:

A1: ya pero tú estás comparando y yo no te pido que compares yo (RISAS) lo que me parece injusto es que digas que no tiene por qué valeer/ y quee- y quee- y que pienses/ y que pienses el asunto de lo del dinero de que sea un curso gratis o no.

B1: no no que no tiene por qué valer el doble es decir

Los hablantes tienen dos opiniones encontradas sobre el tema tratado (los cursos de idiomas), de modo que, en este punto de la conversación, los dos tratan de defender su punto de vista sobre la del otro emitiendo dos intervenciones simultáneamente que no reaccionan entre sí, sino que pretenden hacer prevalecer su voz a través de una lucha por el turno. En este caso, el hablante A gana dicha lucha, puesto que logra que B abandone su intervención para reaccionar a lo que A estaba diciendo.

No obstante, esta figura también puede aparecer en un contexto distinto, cuando los hablantes están totalmente de acuerdo con el tema que se está desarrollando. Es lo que sucede en (14).

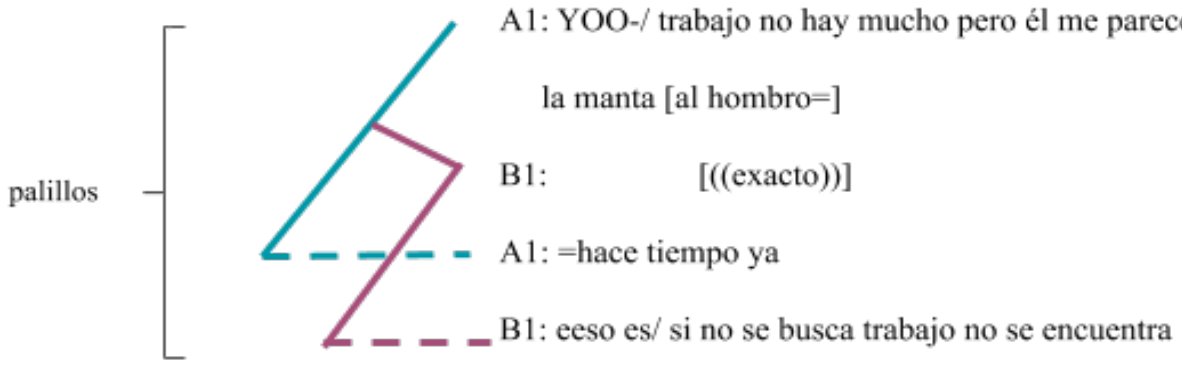


De nuevo los hablantes emiten dos intervenciones discontinuas paralelas entre las cuales, no hay alternancia de turno. Sin embargo, en este ejemplo, los hablantes sí están de acuerdo sobre lo dicho, es más, están tan de acuerdo que lo que provoca la aparición de estos palillos es el alto grado de colaboración e implicación en la conversación por parte de los interlocutores. Sucede que B comparte la opinión de A sobre la falta de esfuerzo, de modo que quiere completar lo dicho por A y, sin querer, se adelanta al final del turno de A1. Es decir, se han producido los palillos por una mala interpretación del LTP. Esto se produce de forma bastante frecuente en la conversación coloquial, pues se trata de un género en el que la distribución de los turnos de habla no está preestablecida. Sin embargo, no se interpretan estos casos como algo inapropiado o invasivo, sino como una muestra de interés y de implicación de los participantes. Se da, por tanto, una breve lucha por el turno, pero no es una lucha conflictiva, como en el caso anterior. Esto hace que este tipo de palillos producidos por una mala interpretación del LTP, se resuelvan rápidamente en pocas intervenciones, de manera que la extensión de estos palillos es mucho más breve que la de aquellos que se producen en una secuencia de desacuerdo (como en el (13).

Es una figura que, tanto en su versión extensa como en la breve, presenta una frecuencia media en la conversación coloquial española (6,60 \%). La participación de los interlocutores en la gestión de esta figura no es equitativa, en tanto que se da una lucha por el turno y el hablante que consigue la reacción final posee un rol dominante. Se produce cuando hay una lucha por el turno, pero dicha lucha puede o no ser conflictiva. La lucha por el turno es conflictiva cuando hay desacuerdo entre los interlocutores, lo cual hace que los palillos se alarguen varios turnos. Su uso abusivo puede ser descortés, puede indicar el dominio del turno de habla de uno de los interlocutores o señalar un desacuerdo argumentativo a causa de algún tema polémico. No es conflictiva, en cambio, cuando no hay desacuerdo, sino que los palillos se producen por una mala interpretación del LTP. Esto hace que dichos palillos sean cortos y, además, se perciben en la conversación coloquial española como cooperativos.

\subsection{Cercos}

Los cercos son figuras complejas asociadas a distintos fenómenos conversacionales que se caracterizan porque en ellas se dan, al menos, dos niveles de recursividad, es decir, son figuras que engloban otras figuras. Según la forma de establecer la conexión entre las intervenciones del nivel principal y las de nivel secundario, podemos distinguir tres tipos: cerco abierto, cerco cerrado y cerco libre.

El cerco cerrado se produce cuando A emite un turno y B reacciona a dicho turno, pero para cerrar ese intercambio, es necesario resolver previamente alguna cuestión que se 
desarrolla mediante una serie de intercambios secundarios. Una vez resuelta esa cuestión,

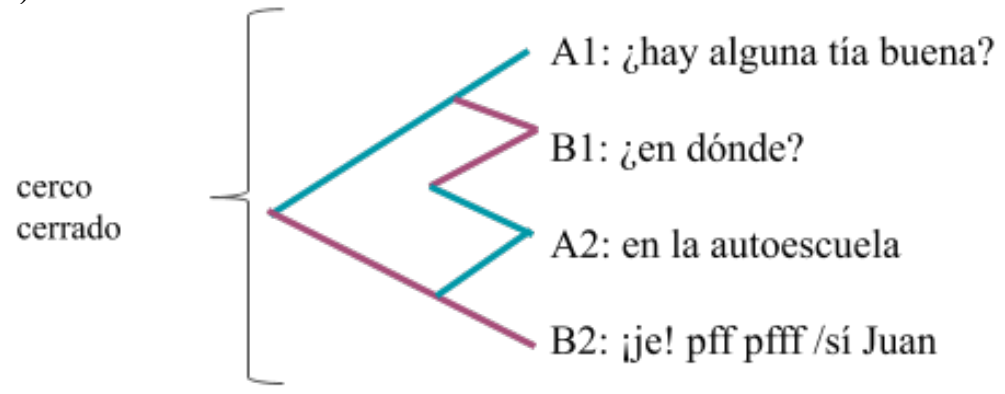

B responde al turno inicial de A cerrando el intercambio marco (ver ejemplo (15).

El rol de los participantes es equitativo, pues los dos intervienen tanto en la gestión del intercambio principal, como en el de los secundarios. Es una figura que presenta una frecuencia escasa en el corpus $(1,2 \%) \mathrm{y}$, como se aprecia en el ejemplo, se emplea para obtener alguna información previa necesaria para responder al turno inicial.

El intercambio principal de un cerco se denomina marco. Se puede encontrar una variante de esta figura, así como del resto de cercos, en la que dicho marco en lugar de estar formado por un intercambio, se forma con una intervención discontinua. Más adelante veremos un ejemplo de esta variante.

El cerco abierto se asemeja al anterior, con la diferencia de que los intercambios del nivel subordinado no son necesarios para resolver el intercambio principal. Es decir, se produce cuando A emite un turno y B reacciona, pero antes de cerrar el intercambio principal, se produce una desviación temática tras la que se vuelve a retomar el diálogo principal. En el ejemplo (16) se da una variante de esta figura en la que el marco del cerco no es un intercambio sino una intervención discontinua.

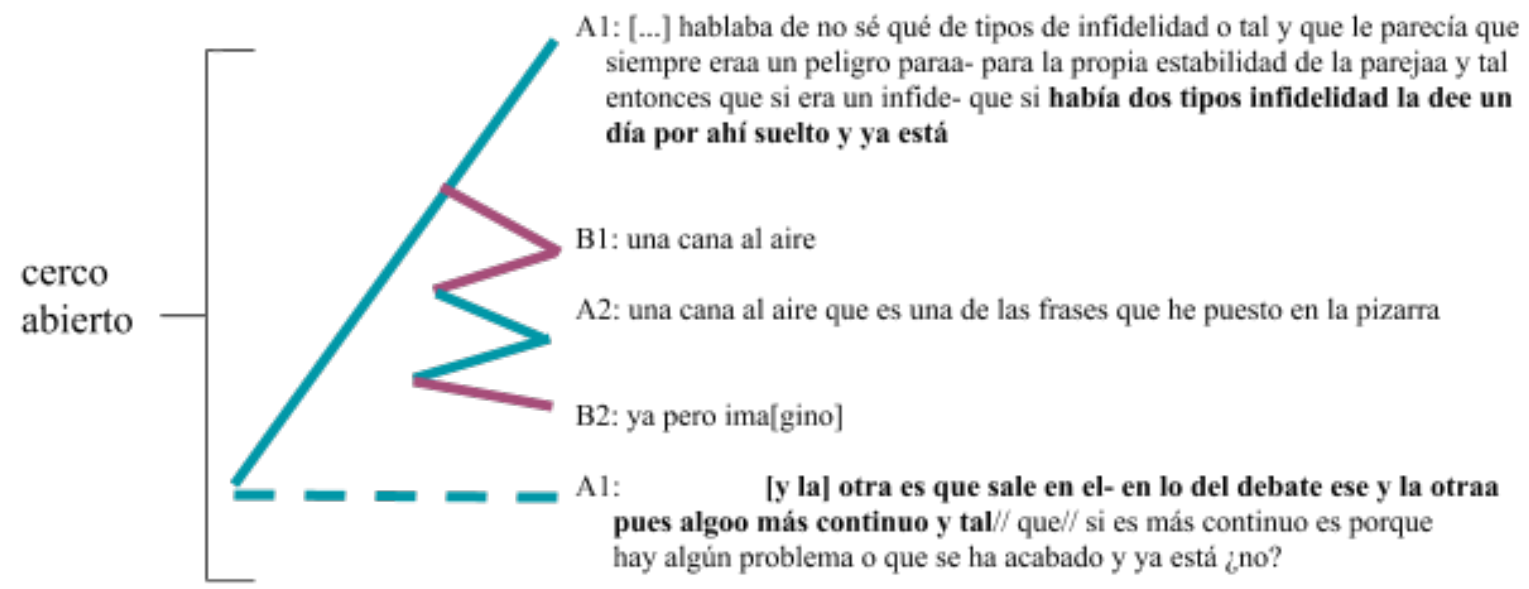

Puesto que el marco de este cerco no está formado por un intercambio sino por una intervención discontinua, el rol de los participantes no es equitativo, en tanto que el hablante A, emisor de la intervención discontinua con función de marco, tiene un papel dominante en este fragmento. Cuando el marco está formado por un intercambio, el rol 
de los participantes sí es equitativo. La figura tiene una frecuencia media en el corpus (5,3 $\%)$ y se suele emplear para aclarar o para aportar información pertinente sobre el tema, aunque no sea estrictamente necesaria. En el ejemplo (16), A1 explica que hay dos tipos de infidelidad, el primero de los cuales es un caso de infidelidad más esporádico. B2 reacciona resumiendo lo dicho por A1 y mostrando que está siguiendo la explicación de su interlocutor. A2 reacciona a $\mathrm{B} 2$ aportando una información complementaria, pertinente para el tema, aunque no imprescindible. B2 reacciona a A1, pero A le interrumpe y le roba el turno sin reaccionar a B2 para continuar con la explicación del segundo tipo de infidelidad, que se trata de un caso más prolongado. Probablemente A corta bruscamente a B2 para evitar perder el hilo o que la conversación se desvíe hacia otros temas, lo cual ilustra su rol dominante en este fragmento.

El último subtipo de cerco, el cerco libre, se relaciona con el anterior en tanto que se produce por una desviación temática, pero se diferencia de aquel porque dicha desviación está totalmente desconectada del diálogo principal. En el ejemplo (17) se puede observar este fenómeno, fruto del contexto comunicativo en el que los hablantes interactúan mientras comen.

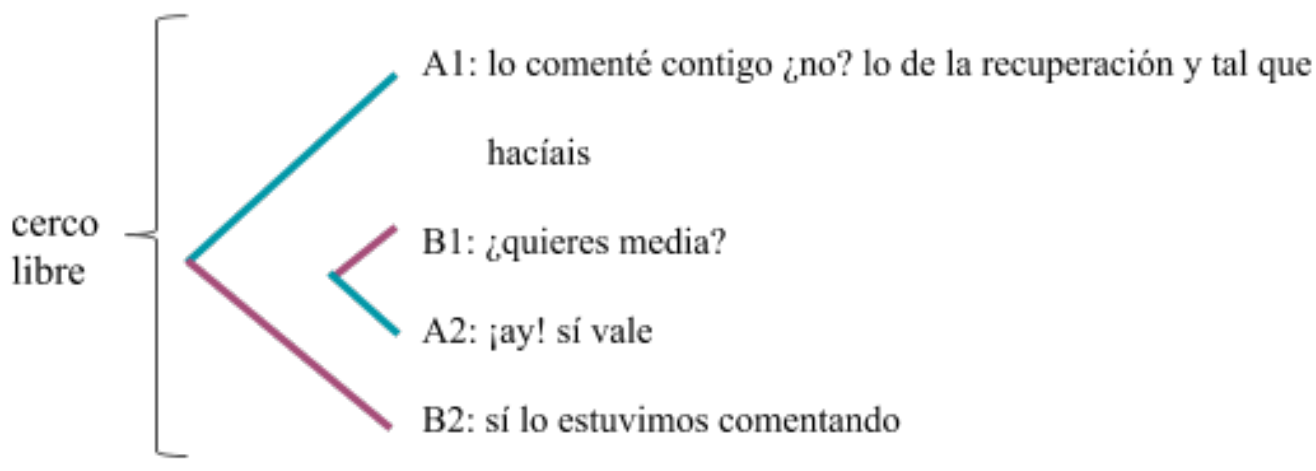

En este fragmento, el diálogo principal trata sobre la recuperación de exámenes. A1 le pregunta a B si ya le ha hablado sobre este tema, pero en lugar de responder a la pregunta en B1, emite una intervención iniciativa en la que realiza una pregunta totalmente desconectada del diálogo principal para resolver una cuestión que tiene que ver con la comida. A2 responde afirmativamente al ofrecimiento de B1, y es entonces cuando B2 contesta a la pregunta inicial de A1 sobre las recuperaciones. En términos del grupo Val.Es.Co., el intercambio mantenido entre B1 y A2 es un diálogo lateral (Grupo Val.Es.Co., 2014: 34). Se trata de un diálogo subordinado al principal pero independiente, puesto que $\mathrm{B} 1$ no reacciona a $\mathrm{A} 1$, ni $\mathrm{B} 2$ reacciona a $\mathrm{A} 2$.

El rol de los interlocutores en esta figura es equitativo. Aparece escasamente en el género de la conversación coloquial $(1,2 \%)$ y se emplea de forma puntual, como hemos visto en (17), para resolver alguna cuestión al margen del diálogo principal, pero sin abandonarlo por completo.

\subsection{Raya y peine}

La raya y el peine son figuras generadas por la aparición de intervenciones independientes, esto es, intervenciones que no reaccionan a nada de lo que se ha dicho previamente ni generan ninguna reacción verbal por parte de los interlocutores. 
La raya es la aparición aislada de una intervención independiente, producida bien por algún elemento del contexto situacional al que reaccionan los hablantes, bien porque el hablante externaliza un sentimiento, sensación o pensamiento en voz alta. Este último caso es el que provoca la aparición de la raya en (18).

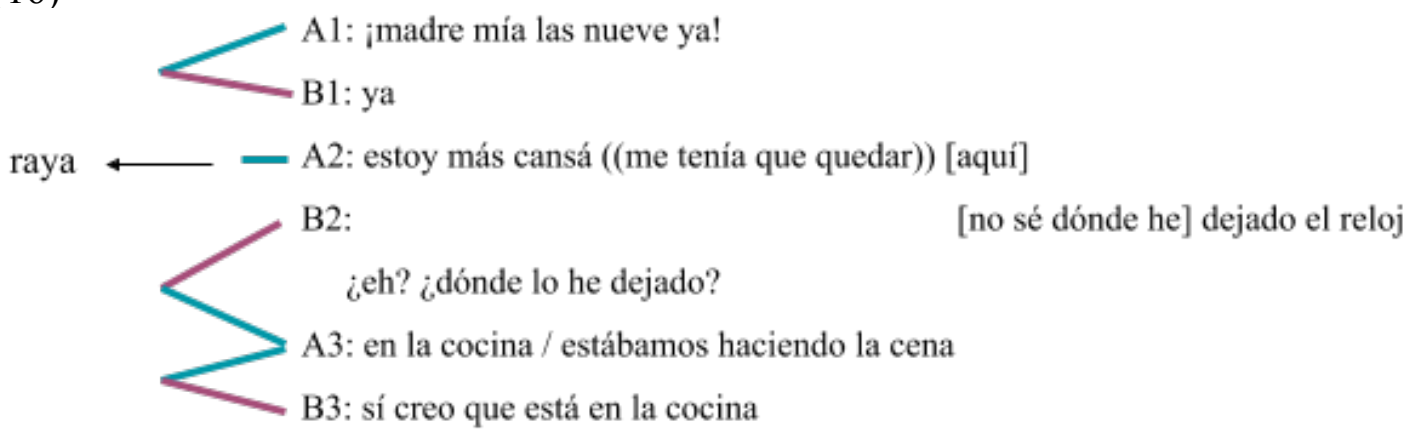

En el diálogo principal que inicia el fragmento, A y B están hablando sobre la hora y, a continuación, A2 exterioriza su estado físico, de modo que no reacciona a B1, sino a un estímulo interno. Asimismo, B2 no reacciona a A2 porque está buscando su reloj y, puesto que no lo encuentra, se lo dice a A para que le ayude.

Es una figura muy poco frecuente en el género $(0,6 \%)$, aunque todavía es más escasa en géneros más formales como la entrevista. El rol de los participantes es equitativo, ya que ningún hablante predomina sobre el otro. Se trata de una figura no dialogal, es decir, los hablantes no reaccionan a lo que ha dicho el otro, sino a otros elementos externos. Su aparición corta el diálogo, de modo que no favorece el desarrollo de los temas.

El peine, por su parte, se produce cuando este fenómeno se prolonga durante más de una intervención, es decir, cuando se producen más de dos intervenciones independientes seguidas (ver ejemplo (19).

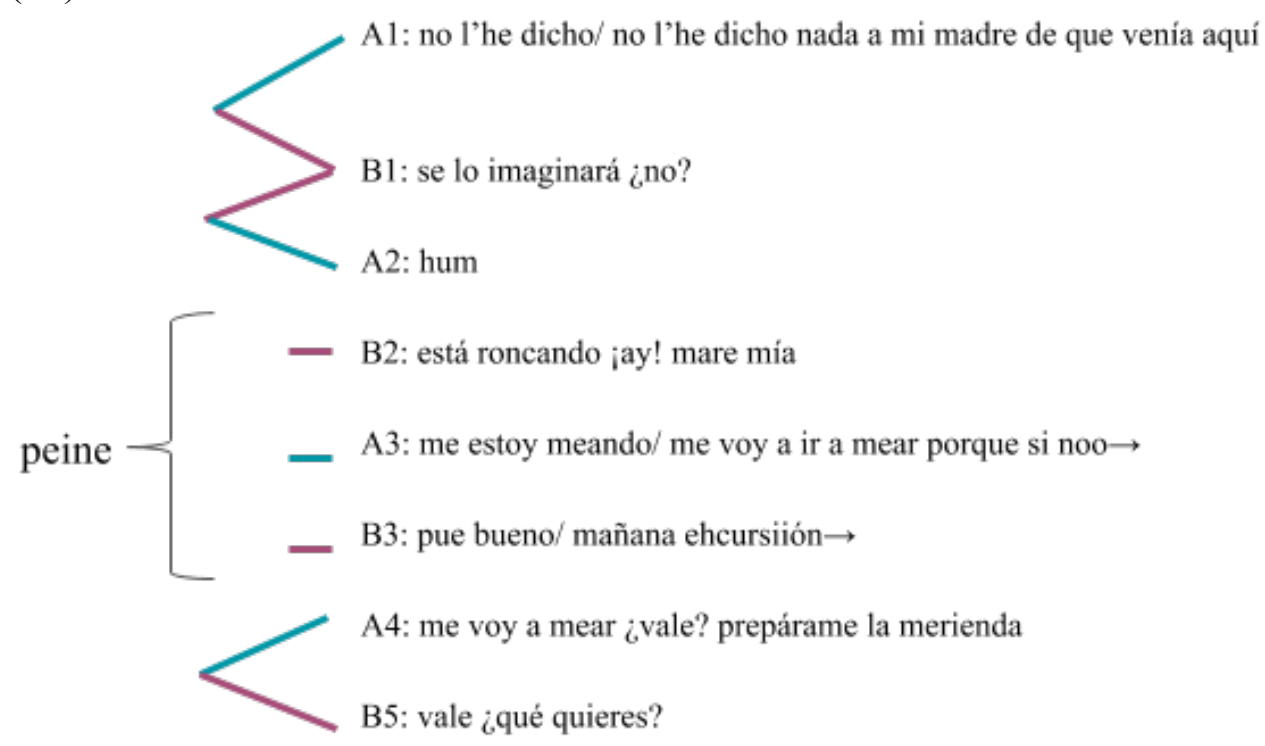

En este fragmento, A y B están hablando sobre informar a la madre de A de que están en casa de $\mathrm{B}$, pero de repente, $\mathrm{B} 2$ reacciona, no a la confirmación de $\mathrm{A} 2$ sino al perro que 
está a su lado y ha empezado a roncar. Sin embargo, A3 no reacciona a este nuevo tema, sino que emite una nueva intervención independiente que reacciona a un estímulo interno. Lo mismo sucede en B3 cuando el hablante se acuerda de que al día siguiente tiene una excursión y lo dice en voz alta. No obstante, A quiere que B le prepare la merienda mientras va al baño, de modo que repite lo que había dicho en $\mathrm{A} 3 \mathrm{y}$ le pide expresamente a B que le haga la merienda, a lo cual sí que reacciona B5 de forma afirmativa.

Esta figura es muy escasa en la conversación coloquial (1,6\%), aunque es una estructura propia del registro coloquial y no suele darse en otros registros más formales. Esto es así debido al anclaje de la conversación coloquial al contexto situacional, rasgo menos marcado en otros géneros como la entrevista. Se produce, como se ha podido comprobar en los ejemplos, cuando un hablante reacciona a un estímulo interno (sueño, hambre, dolor, cansancio, etc.) y lo externaliza verbalmente, o cuando reacciona a algún elemento del contexto situacional. El rol de los participantes, al igual que en la raya, es equitativo, y se trata de una figura no dialogal. Es una estructura, por tanto, que fragmenta el diálogo $\mathrm{y}$, aunque su aparición en la conversación es natural, su uso excesivo puede impedir el desarrollo de los temas, porque su presencia prolongada indica que algo externo al diálogo y perteneciente al contexto situacional está irrumpiendo en la conversación.

\section{Propuesta didáctica para enseñar las estructuras interaccionales en el aula de ELE}

Con el fin de ilustrar cómo se pueden aplicar a la enseñanza de ELE los dientes de sierra y la tipología de estructuras interaccionales, se propone a continuación una secuencia didáctica cuyos objetivos son:

- Familiarizar a los alumnos con todo el abanico de estructuras interaccionales que tienen a su alcance.

- Dar a conocer las estrategias pragmáticas que subyacen a cada una de esas estructuras.

- Mostrar cómo se pueden emplear las diferentes figuras para mejorar la competencia conversacional.

Una secuencia como esta puede ayudar a minimizar los malentendidos y fallos de comunicación que se producen cuando hablantes extranjeros conversan con nativos a causa de las diferencias que rigen la alternancia de turnos de su lengua de origen con respecto a la de la lengua meta comentados en el marco teórico.

La propuesta titulada Dibuja la conversación está dirigida a alumnos de nivel B2 o superior y en ella se trabaja la comprensión oral y escrita. Para su desarrollo es necesario disponer de un dispositivo reproductor de audio y de un dosier con los siguientes contenidos teóricos: 1) presentación de los dientes de sierra y de su funcionamiento básico; 2) tipología de figuras interaccionales (de forma similar a como se ha presentado en este trabajo ${ }^{6}$ ); 3) ficha-resumen de las funciones pragmáticas asociadas a cada figura con ejemplos ilustrativos de todas ellas; 4) Inventario de los turnos de apoyos verbales y no verbales; y 5) transcripción de fragmentos seleccionados de conversaciones

\footnotetext{
${ }^{6}$ Ver tabla Tabla 2.
} 
coloquiales $^{7}$. El tiempo total estimado para realizar la propuesta es de cuatro sesiones de hora y media cada una.

La secuencia consta de tres partes. La primera parte (sesión 1) sirve para introducir el tema y activar conocimientos previos a partir de dos actividades. La primera actividad consiste en un pequeño diálogo para todo el grupo a partir de las siguientes preguntas: ¿Cómo conversas en tu lengua cuando hablas con amigos o familiares?, ¿has detectado alguna diferencia con respecto a cómo conversan coloquialmente los españoles?, ¿en qué aspectos lo has notado? En la segunda actividad, pedimos a los alumnos que elaboren un listado con aquello que deben hacer los interlocutores en una conversación coloquial para ser cooperativos y corteses, y lo que hace que no lo sean. Algunos ejemplos del tipo de rasgos que pueden aportar los alumnos se pueden apreciar en la Tabla 3.

\begin{tabular}{|c|c|}
\hline $\begin{array}{l}\text { Rasgos cooperativos y } \\
\text { corteses }\end{array}$ & $\begin{array}{l}\text { - } \text { Mirar al interlocutor } \\
\text { - No interrumpir } \\
\text { - Asentir con la cabeza } \\
\text { - Dejar hablar al otro, hacerle preguntas }\end{array}$ \\
\hline $\begin{array}{l}\text { Rasgos no cooperativos y } \\
\text { descorteses }\end{array}$ & $\begin{array}{l}\text { - Mirar el móvil mientras el interlocutor habla } \\
\text { - Interrumpir constantemente } \\
\text { - No mostrar ninguna reacción a lo que dice el } \\
\text { interlocutor } \\
\text { - No dejar hablar al otro, no hacer ninguna } \\
\text { pregunta }\end{array}$ \\
\hline
\end{tabular}

Tabla 3. Rasgos interaccionales que favorecen la cooperación en la conversación coloquial.

Esta parte introductoria sirve para que los alumnos compartan y sean conscientes de las reglas socioculturales que dominan la toma de turnos de las diferentes lenguas y que provocan malentendidos en la comunicación. Una vez hecho esto explicamos, con ayuda del dosier, en qué consisten los dientes de sierra, cómo se emplea dicha herramienta, así como la tipología de estructuras y la ficha-resumen de las figuras.

La segunda parte (sesión 2 y 3 ) de la secuencia consta de tres actividades. En la primera de ellas pedimos a los alumnos que escuchen una conversación coloquial y dibujen con los dientes de sierra la relación entre los turnos sobre uno de los fragmentos incluidos en el dosier ${ }^{8}$. En la segunda actividad, los alumnos deben identificar las estructuras conversacionales que han aparecido en el fragmento. Esta actividad se puede hacer individualmente o por parejas, y una vez finalizada, se puede poner en común y comparar los análisis. La tercera y última actividad de esta parte consiste en observar y comentar los siguientes aspectos de la conversación analizada:

\footnotetext{
${ }^{7}$ Los fragmentos de conversaciones coloquiales se pueden seleccionar del corpus Val.Es.Co. 2.0 (Cabedo y Pons, 2013) o emplear los ejemplos proporcionados en este mismo artículo.

${ }^{8}$ En el siguiente enlace se pueden consultar los fragmentos para analizar junto con el solucionario: https://docs.google.com/document/d/1lb98BNJJnGIISGO7zEgqHmAE0WzPiKo3sNY-

RTRU7Qs/edit?usp=sharing.
} 
a. ¿Se producen todas las figuras estudiadas?, ¿cuáles son las más abundantes?, ¿cuáles las que menos?

b. ¿Hay algún hablante que domine la toma de turnos?, ¿hay algún hablante más pasivo?

c. ¿Es una conversación cooperativa y cortés o hay alguna disputa tensa?, en este caso, ¿qué figuras se dan en el conflicto?

d. ¿Es una conversación fragmentada o cohesionada y dinámica?, ¿qué figuras indican esto?

Por último, la tercera parte de la secuencia (sesión 4) se centra en la reflexión sobre su propia actividad conversacional en español. Una vez los alumnos conocen en mayor profundidad cómo funciona la toma de turnos del español, están en condiciones de identificar qué aspectos deberían mejorar. Para orientar esta reflexión, facilitamos las siguientes preguntas que los alumnos deberán plantearse individualmente:

a. ¿Tiendes a ocupar la mayoría de los turnos?, ¿tienes un rol pasivo?, ¿tratas de conseguir que haya una igualdad participativa entre los interlocutores?

b. ¿Eres cooperativo?, ¿favoreces el desarrollo de los temas?, ¿qué figuras podrías emplear para mejorar en este aspecto?

c. ¿Eres cortés?, ¿con qué estructuras lo consigues?, ¿deberías emplear más alguna de las estructuras estudiadas para ser más cortés?, ¿qué estructuras debes evitar para no serlo?

d. ¿Favoreces la cohesión de la conversación?, ¿cómo lo haces?, ¿cómo podrías mejorar este aspecto?, ¿qué debes evitar para no fragmentar el diálogo?

\section{Conclusiones}

Con el presente trabajo se ha puesto de manifiesto la necesidad, ya apuntada por otras autoras (Cestero Mancera, 2012; García García, 2016), de sensibilizar a los estudiantes de español sobre las diferencias existentes en la toma de turnos de la conversación coloquial española. Para alcanzar dicho objetivo se proponen los dientes de sierra como herramienta para facilitar un acercamiento didáctico a las estructuras conversacionales gracias a su carácter visual y esquemático. Asimismo, a través de las figuras descritas en la tipología de estructuras conversacionales (Espinosa-Guerri y García-Ramón, 2019), se pueden enseñar estrategias pragmáticas asociadas a la producción de determinadas estructuras como, por ejemplo, el dinamismo interaccional, los turnos de apoyo verbales y no verbales, el desarrollo de temas, los cambios de tópico, la cohesión discursiva, etc. Una secuencia didáctica diseñada, por tanto, a partir de los dientes de sierra y la tipología de estructuras puede ayudar a los estudiantes de español a ser más conscientes de los rasgos de la conversación coloquial española, de las diferencias con respecto a su lengua de origen y de los recursos que deben emplear para mejorar la competencia conversacional. Asimismo, en la línea que propone Padilla García (2012), el conocimiento de las diferentes figuras que se pueden dar en la conversación coloquial española puede ser útil para los creadores de materiales con el fin de ofrecer muestras de habla de la conversación coloquial más ajustadas a la realidad.

\section{Bibliografía}

BRIZ GóMEZ, Antonio y Grupo Val.Es.Co. (2003). «Las unidades de la conversación: el acto». En J. L. Girón (Ed.); Estudios ofrecidos al profesor José Jesús de Bustos Tovar: 
Vol. II (pp. 953-968). Madrid: Universidad Complutense.

BRIZ GÓMEZ, Antonio. (2006). «La segmentación de una conversación en diálogos», Oralia: Análisis del discurso oral, (9), 45-72.

BRIz GÓMEZ, A. (2010). «Lo coloquial y lo formal, el eje de la variedad lingüística». De moneda nunca usada: Estudios dedicados a José Ma Enguita Utrilla, 2010, (pp. 125133). Recuperado el 27/04/2019 de: https://dialnet.unirioja.es/servlet/articulo?codigo= 3923618

Cestero Mancera, Ana María (2000). Los turnos de apoyo conversacionales. Cádiz: Universidad de Cádiz.

Cestero Mancera, Ana María (2012). «La enseñanza de la conversación en ELE: Estado de la cuestión y perspectivas de futuro», Revista Internacional de Lenguas Extranjeras / International Journal of Foreign Languages, 0(1), 31. Recuperado el 27/04/2019 de: https://doi.org/10.17345/rile201231-62

Cestero MAnCERA, Ana María (2016). «La conversación. Bases teóricas y metodológicas para su investigación y su enseñanza en ELE», Linred: Lingüística en la Red, (14), 2.

ESPINOSA-GUERRI, Guadalupe (2016). «Dientes de sierra: una herramienta para el estudio de la estructura interactiva del discurso dialógico», Normas, 6, 13-28.

ESPINOSA-GUERRi, Guadalupe y GARCÍA-RAMÓN, Amparo (2017). «Conversational structures and discourse genres: a contrastive study of informal conversation, sociolinguistic interviews and broadcast interviews», 15th International Pragmatics Conference, Belfast, 16-21/07/2017.

ESPINOSA-GUERRi, Guadalupe y GARCÍA-RAMÓN, Amparo (2019). «Visualizing conversational structure: a preliminary typology of interactional figures». En Ó. Loureda Lamas, I. Recio Fernández, L. Nadal y A. Cruz (Eds.); Empirical Approaches to the Construction of Discourse: Information Structures, Argumentative Relations and Discourse Units. Amsterdam/Philadelphia: John Benjamins.

GARCÍA GARCÍA, Marta (2016). «La alternancia de turnos y la organización temática en la conversación entre estudiantes alemanes de ELE», Linred: Lingüistica en la Red, (14), 3.

PADilla García, Xose (2012). «¿Qué tienen de 'conversación coloquial' los diálogos que aparecen en los libros de ELE?», Verba: Anuario Galego de Filoloxía, 39(0). Recuperado el 27/04/2019 de: http://www.usc.es/revistas/index.php/verba/article/ view/870

GRUPO VAL.Es.CO. (2014). «Las unidades del discurso oral: la propuesta Val.Es.Co. de segmentación de la conversación (coloquial)», Estudios de lingüística del español, 35, 11-71.

INGLÉS CANDELAS, Beatriz. (2016). «El funcionamiento de los turnos de apoyo en la conversación en ELE de estudiantes ingleses», Linred: Lingüistica en la Red, (14), 6.

SCHEGLOFF, Emanuel (2007). Sequence Organization in Interaction: Volume 1: A Primer in Conversation Analysis. Cambridge University Press.

Stivers, T., Enfield, N. J., Brown, P., et al. (2009). «Universals and cultural variation 
in turn-taking in conversation», Proceedings of the National Academy of Sciences, 106(26), pp. 10587-10592. Recuperado el 27/04/2019 de: https://www.academia.edu /12884850/Universals_and_cultural_variation_in_turn-taking_in_conversation. 